\title{
The Neumann Type Boundary Value Problem in the Theory of Thermoelasticity with Microtemperatures for a Plane with Circular Hole
}

\author{
Lamara Bitsadze \\ I. Vekua Institute of Applied Mathematics of IV. Javakhishvili Tbilisi State University, 2 University St., 0186 Tbilisi, Georgia
}

\section{Keywords}

Thermoelasticity with microtemperatures, Explicit solution, Plane with circular hole.

\begin{abstract}
This paper studies the linear theory of thermoelastic materials with inner structure whose particles,in addition to the classical displacement and temperature fields, possess microtemperatures. The present work considers the 2D equilibrium theory of thermoelasticity for solids with microtemperatures. This paper is devoted to the explicit solution of the Neumann type boundary value problem for an elastic plane, with microtemperatures having a circular hole. Special representations of the regular solutions of the considered equations are constructed by means of the elementary (harmonic, biharmonic and meta-harmonic) functions. Using the Fourier method, we presented the solution of the Neumann type boundary value problem for the plane with circular hole in the explicit form.
\end{abstract}

\section{Introduction}

The linear theory of thermoelasticity for materials with inner structure whose particles, in addition to the classical displacement and temperature fields, possess microtemperatures was established by Grot [1]. Ieşan and Quintanilla in [2] considered the dynamical theory of homogeneous and isotropic thermoelastic bodies, have formulated the boundary value problems and presented an uniqueness theorems, also they established a counterpart of the Boussinesq-Somigliana-Galerkin solution in the classical elastostatics. The fundamental solutions of the equations of the three-dimensional (3D) theory of thermoelasticity with microtemperatures were constructed by Svanadze in [3]. The representations of the Galerkin type and general solutions of the system of static of the above mentioned theory were obtained by Scalia, Svanadze, and Tracina [4]. The linear theory of micromorphic elastic solids with microtemperatures was considered by Ieşan [5].

For applications, it is especially important to construct the solutions boundary value problems (BVPs) in explicit form because such solutions enable us to effectively perform quantitative analysis of the investigated problems. Some works of the $2 \mathrm{D}$ and $3 \mathrm{D}$ theories of elasticity for materials with microstructures can be seen in [6-30], in which give the main results and bibliographical data and also the explicit solutions are constructed for some BVPs for the concrete domains.

The present paper considers the $2 \mathrm{D}$ equilibrium theory of thermoelasticity for solids with microtemperatures. The present article is arranged as follows: in Section 2, the basic equations are presented in terms of the displacement vector, temperature and the microtemperatures. The basic BVP is formulated for a plane with a circular hole. In Section 3, the regular solutions of the considered equations are given by means of the elementary (harmonic, biharmonic and meta-harmonic) functions. In Section 4 the Neumann type BVP is solved explicitly for a plane with a circular hole. The obtained solutions are given in the explicit form.
2. Basic Equations. Boundary value problems

Let $x=\left(x_{1}, x_{2}\right)$ be a point in the Euclidean two-dimensional space $E^{2}$. Let $D^{-}$be the whole plane with a circular hole, bounded by the circumference $S$ with center at the origin and radius $R$. Let us assume that the domain $D^{-}$is filled with an isotropic thermoelastic material with micro-temperatures.

The governing homogeneous system of the theory of thermoelasticity with microtemperatures has the form [3]

$\mu \Delta \mathbf{u}+(\lambda+\mu) \operatorname{graddiv} \mathbf{u}-\operatorname{grad}(\beta \theta)=0$,

$k \Delta \theta+k_{1} \operatorname{div} \mathbf{w}=0$ $k_{6} \Delta \mathbf{w}+\left(k_{4}+k_{5}\right)$ graddiv $\mathbf{w}-k_{3} \operatorname{grad} \theta-k_{2} \mathbf{w}=0$ where $\mathbf{u}(\mathbf{x})=\left(u_{1}, u_{2}\right)^{T}$ is the displacement vector in a solid, $\mathbf{W}=\left(w_{1}, w_{2},\right)^{T}$ is called microtemperatures vector, $\theta$ is the temperature measured from the constant absolute temperature $T_{0}\left(T_{0}>0\right)$, of the natural state, $k_{j}, k, \lambda, \mu, \beta$ are the constitutive coefficients, $\Delta$ is the 2D Laplace operator. superscript " $T$ " stands for the transpose operation.

Definition. A vector-function $\mathbf{U}=(\mathbf{u}, \mathbf{w}, \theta)^{T}$ defined in the domain $D^{-}$is called regular if $\mathbf{U} \in C^{2}\left(D^{-}\right) \cap C^{1}\left(\overline{D^{-}}\right)$and 
the vector $\mathbf{U}$ should additionally satisfy the following conditions at infinity:$$
\mathbf{U} \in O\left(|\mathbf{x}|^{-1}\right), \quad \frac{\partial \mathbf{U}}{\partial x_{j}} \in O\left(|\mathbf{x}|^{-2}\right), \quad j=1,2,
$$

$$
|\mathbf{x}|^{2}=x_{1}^{2}+x_{2}^{2}
$$

For the equations (1)-(3) we consider the following basic BVP.

Problem 1. Find a regular solution $\mathbf{U}$ to the equations (1)-(3) in the domain $D^{-}$, satisfying the following boundary conditions on $S$ :

$(\mathbf{P U})^{-}=\mathbf{f}^{-}(\mathbf{z}), \quad \mathbf{w}^{-}=\mathbf{F}^{-}(\mathbf{z}), \quad \theta^{-}=f_{3}^{-}(\mathbf{z})$, $z \in S$,

where $(\cdot)^{-}$denotes the limits of vector $\mathbf{V}=(\mathbf{P u}, \mathbf{w}, \theta)^{T}$ on $\mathbf{z} \in S$ from $D^{-}$

$\mathbf{V}^{-}(\mathbf{z})=\lim _{D^{-} \ni x \rightarrow z \in S} \mathbf{V}(\mathbf{x})$

$\mathbf{f}\left(f_{1}, f_{2}\right), \quad \mathbf{F}\left(F_{1}, F_{2}\right)$ and $f_{3}$ are the given functions. Moreover we assume that the boundary values are absolute integrable functions. The vector $\mathbf{P}\left(\partial_{x}, \mathbf{n}\right) \mathbf{U}$ is defined in the following form

$\mathbf{P}\left(\partial_{x}, \mathbf{n}\right) \mathbf{U}=\mathbf{T}\left(\partial_{x}, \mathbf{n}\right) \mathbf{u}-\mathbf{n}(\beta \theta)$,

$\mathbf{T}\left(\partial_{x}, \mathbf{n}\right) \mathbf{u}$ is the stress vector in the classical theory of elasticity

$\mathbf{T}\left(\partial_{x}, \mathbf{n}\right) \mathbf{u}=2 \mu \frac{\partial \mathbf{u}}{\partial n}+\lambda \mathbf{n} \operatorname{div} \mathbf{u}+\mu\left(\begin{array}{c}n_{2} \\ -n_{1}\end{array}\right)\left(\frac{\partial u_{2}}{\partial x_{1}}-\frac{\partial u_{1}}{\partial x_{2}}\right)$ $\mathbf{n}\left(n_{1}, n_{2}\right)$ is a normal vector, and $\frac{\partial}{\partial n}$ is the derivative along the vector $\mathbf{n}\left(n_{1}, n_{2}\right)$.

3. Representation of regular solutions of the system (1)-(3)

In [10] it is proved that the regular solution of the system (2),(3) can be expresented in the form

$\mathbf{w}=-\operatorname{grad}\left[\frac{k_{3}}{k_{2}} \vartheta+\frac{k}{k_{1}} \vartheta_{1}\right]+\mathbf{w}$

$\stackrel{2}{\mathbf{w}}=-\frac{k_{6}}{k_{2}} \operatorname{rotrot} \mathbf{w}, \quad \theta=\vartheta+\vartheta_{1}$,

where $\Delta \vartheta=0, \operatorname{div}{ }^{2} \mathbf{w}=0$

$\left(\Delta-s_{1}^{2}\right) \vartheta_{1}=0, \quad\left(\Delta-s_{2}^{2}\right)^{2} \mathbf{w}=0, \quad s_{2}^{2}=\frac{k_{2}}{k_{6}}>0$ $\operatorname{div} \mathbf{w}=-\frac{k}{k_{1}} s_{1}^{2} \vartheta_{1}, \quad s_{1}^{2}=\frac{k k_{2}-k_{1} k_{3}}{k^{*} k_{7}}>0$.

Let us assume that $\theta$ is known functions when $\mathbf{X} \in D^{-}$. Then from (1), after obvious transformations, for $\mathbf{u}(\mathbf{x})$ and $\operatorname{div} \mathbf{u}$ we obtain the following non-homogeneous equations

$\Delta \operatorname{div} \mathbf{u}=\frac{\beta s_{1}^{2}}{\mu_{0}} \vartheta_{1}, \quad \mu_{0}=\lambda+2 \mu$

$\Delta \mathbf{u}=-\operatorname{grad}\left[\frac{\lambda+\mu}{\mu} \psi-\frac{\beta}{\mu} \vartheta-\frac{\beta}{\mu_{-}} \vartheta_{1}\right]$

solutions of which can be written as

$\operatorname{div} \mathbf{u}=\psi+\frac{\beta}{\mu_{0}} \vartheta_{1}$

$\mathbf{u}=\boldsymbol{\Psi}-\operatorname{grad}\left[\frac{\lambda+\mu}{\mu} \psi_{0}-\frac{\beta}{\mu} \vartheta_{0}-\frac{\beta}{\mu_{0} s_{1}^{2}} \vartheta_{1}\right]$

where $\boldsymbol{\Psi}$ and $\psi$ are an arbitrary harmonic functions $\Delta \boldsymbol{\Psi}=0$, $\Delta \psi=0$, the functions $\vartheta, \psi, \vartheta_{0}, \psi_{0} \quad$ and $\boldsymbol{\Psi}$ are interrelated by the following relations:

$\Delta \vartheta_{0}=\vartheta, \quad \Delta \psi_{0}=\psi, \quad \operatorname{div} \boldsymbol{\Psi}=\frac{\mu_{0}}{\mu} \psi-\frac{\beta}{\mu} \vartheta$

From the above reasoning we have proved the following theorem:

Theorem 3 The regular solution $\mathbf{U}=(\mathbf{u}, \mathbf{w}, \theta)^{T}$ of equations (1)-

(3) admits in the domain $D$ a representation

$\mathbf{u}=\Psi-\operatorname{grad}\left[\frac{\lambda+\mu}{\mu} \psi_{0}-\frac{\beta}{\mu} \vartheta_{0}-\frac{\beta}{\mu_{0} s_{1}^{2}} \vartheta_{1}\right]$.

$\mathbf{w}=-\operatorname{grad}\left[\frac{k_{3}}{k_{2}} \vartheta+\frac{k}{k_{1}} \vartheta_{1}\right]+\stackrel{2}{\mathbf{w},} \quad \stackrel{2}{\mathbf{w}}=-\frac{k_{6}}{k_{2}} \operatorname{rotrot} \mathbf{w}$,

$\theta=\vartheta+\vartheta_{1}, \operatorname{div} \boldsymbol{\Psi}=\frac{\mu_{0}}{\mu} \psi-\frac{\beta}{\mu} \vartheta$,

$\operatorname{div} \mathbf{u}=\psi+\frac{\beta}{\mu_{0}} \vartheta_{1}, \quad \operatorname{div} \mathbf{w}=-\frac{k}{k_{1}} s_{1}^{2} \vartheta_{1}$.

\section{Explicit solution of the Problem 1}

Let us introduce the polar coordinates:

$x_{1}=\rho \cos \eta, \quad x_{2}=\rho \sin \eta$,

$\rho=\sqrt{x_{1}^{2}+x_{2}^{2}}, \quad 0 \leq \eta \leq 2 \pi$.

Note that in 2D space " rot " is defined as a scalar 
$\operatorname{rot} \Phi=\frac{\partial \Phi_{2}}{\partial x_{1}}-\frac{\partial \Phi_{1}}{\partial x_{2}}$

for a vector $\Phi=\left(\Phi_{1}, \Phi_{2}\right)$ and as a vector

$\operatorname{rot} \psi=\left(\frac{\partial \psi}{\partial x_{2}},-\frac{\partial \psi}{\partial x_{1}}\right) \quad$ for a scalar $\psi$.

The following identities are holds:

$\frac{\partial}{\partial n} \operatorname{grad} g=\frac{1}{\rho} \operatorname{grad}\left(\rho \frac{\partial}{\partial \rho}-1\right) g$,

$\operatorname{div} \mathbf{n} g=\left(\frac{\partial}{\partial \rho}+\frac{1}{\rho}\right) g$

$\operatorname{div} \frac{\partial \mathbf{u}}{\partial n}=\left(\frac{\partial}{\partial \rho}+\frac{1}{\rho}\right) \operatorname{div} \mathbf{u}-\frac{1}{\rho}\left(\mathbf{n} \cdot \frac{\partial \mathbf{u}}{\partial n}\right)=$

$\left(\frac{\partial}{\partial \rho}+\frac{1}{\rho}\right) \operatorname{div} \mathbf{u}-\frac{1}{\rho^{2}}\left(\mathbf{x} \cdot \frac{\partial \Psi}{\partial n}\right)$

$+\frac{1}{\rho} \frac{\partial^{2}}{\partial \rho^{2}}\left[\frac{\lambda+\mu}{\mu} \psi_{0}-\frac{\beta}{\mu} \vartheta_{0}-\frac{\beta}{\mu_{0} s_{1}^{2}} \vartheta_{1}\right]$,

$\operatorname{div}\left(\begin{array}{c}n_{2} \\ -n_{1}\end{array}\right)\left(\frac{\partial \Psi_{2}}{\partial x_{1}}-\frac{\partial \Psi_{1}}{\partial x_{2}}\right)=-\frac{\partial}{\partial \rho} \operatorname{div} \Psi$,

if $\Delta \Psi=0$,

$\left(\mathbf{x} \cdot \frac{\partial \Psi}{\partial n}\right)=\left(\frac{\partial}{\partial n}-\frac{1}{\rho}\right)(\mathbf{x} \cdot \Psi)$

$\lambda \operatorname{div} \mathbf{u}-\beta \theta=\lambda \psi-\beta \vartheta-\frac{2 \mu \beta}{\mu_{0}} \vartheta_{1}$.

Using these identities, the stress vector takes the form

$\mathbf{P}\left(\partial_{x}, \mathbf{n}\right) \mathbf{U}=2 \mu \frac{\partial \Psi}{\partial n}$

$\frac{2 \mu}{\rho} \operatorname{grad}\left(\rho \frac{\partial}{\partial \rho}-1\right)\left[\frac{\lambda+\mu}{\mu} \psi_{0}-\frac{\beta}{\mu} \vartheta_{0}-\frac{\beta}{\mu_{0} s_{1}^{2}} \vartheta_{1}\right]$ (7)

$+\mathbf{n}[\lambda \operatorname{div} \mathbf{u}-\beta \theta]+\mu\left(\begin{array}{c}n_{2} \\ -n_{1}\end{array}\right)\left(\frac{\partial \Psi_{2}}{\partial x_{1}}-\frac{\partial \Psi_{1}}{\partial x_{2}}\right)$.

From (4), (6)and (7), by direct calculation, we readily get: $(\mathbf{x} \cdot \mathbf{P u})=2 \mu\left(\mathbf{x} \cdot \frac{\partial \Psi}{\partial n}\right)$

$-2 \mu \rho \frac{\partial^{2}}{\partial \rho^{2}}\left[\frac{\lambda+\mu}{\mu} \psi_{0}-\frac{\beta}{\mu} \vartheta_{0}-\frac{\beta}{\mu_{0} s_{1}^{2}} \vartheta_{1}\right]$

$+\rho[\lambda \operatorname{div} \mathbf{u}-\beta \theta]$

$\operatorname{div} \mathbf{P} \mathbf{u}=-\frac{2 \mu}{\rho^{2}}\left(\mathbf{x} \cdot \frac{\partial \Psi}{\partial n}\right)$

$+\frac{2 \mu \partial^{2}}{\rho \partial \rho^{2}}\left[\frac{\lambda+\mu}{\mu} \psi_{0}-\frac{\beta}{\mu} \vartheta_{0}-\frac{\beta}{\mu_{0} s_{1}^{2}} \vartheta_{1}\right]$ (8)

$+\frac{\mu_{0} \psi-\beta \vartheta}{\rho}$,

$\frac{1}{\rho^{2}}(\mathbf{x} \cdot \mathbf{P u})+\operatorname{div} \mathbf{P u}=$

$\frac{2}{\rho}\left((\lambda+\mu) \psi-\frac{\mu \beta}{\mu_{0}} \vartheta_{1}-\beta \vartheta\right)$

$(\mathbf{x} \cdot \mathbf{w})=-\rho \frac{\partial}{\partial \rho}\left[\frac{k_{3}}{k_{2}} \vartheta+\frac{k^{*}}{k_{1}^{*}} \vartheta_{1}\right]+(\mathbf{x} \cdot \mathbf{w})$,

$\theta=\vartheta+\vartheta_{1}, \quad \operatorname{div} \mathbf{w}=-\frac{k}{k_{1}} s_{1}^{2} \vartheta_{1}$

$\left(\Delta-s_{2}^{2}\right)(\mathbf{x} \cdot \mathbf{w})=0$

where $(\mathbf{x} \cdot \mathbf{u})$ denotes the scalar product of two vectors.

A solution of Problem 1 is sought in the domain $D^{-}$in the form (6), where the harmonic and the meta-harmonic functions

$\vartheta, \quad \psi, \quad \vartheta_{1}, \quad$ and $(\mathbf{x} \cdot \mathbf{w})$ can be represented as follows

$\vartheta(\mathbf{x})=\frac{E_{0}}{2}+\sum_{n=1}^{\infty}\left(\frac{R}{\rho}\right)^{n}\left(E_{n} \cos n \eta+M_{n} \sin n \eta\right)$,

$\psi(\mathbf{x})=\frac{A_{0}^{\prime}}{2}+\sum_{n=1}^{\infty}\left(\frac{R}{\rho}\right)^{n}\left(A_{n}^{\prime} \cos n \eta+B_{n}^{\prime} \sin n \eta\right)$,

$\vartheta_{1}=\frac{C_{10}}{2} \frac{H_{0}^{(1)}\left(i s_{1} \rho\right)}{H_{0}^{(1)}{ }_{0}\left(i s_{1} R\right)}$

$+\sum_{n=1}^{\infty} \frac{H_{n}^{(1)}\left(i s_{1} \rho\right)}{H_{n}^{(1)}\left(i s_{1} R\right)}\left(C_{1 n} \cos n \eta+D_{1 n} \sin n \eta\right)$, 
$\left(\begin{array}{c}\mathbf{2} \\ \mathbf{x} \cdot \mathbf{w}\end{array}\right)=\frac{C_{10}^{\prime \prime}}{2} \frac{H_{0}^{(1)}\left(i s_{2} \rho\right)}{H_{0}^{(1)}\left(i s_{2} R\right)}+$

$\sum_{n=1}^{\infty} \frac{H_{n}^{(1)}\left(i s_{2} \rho\right)}{H_{n}^{(1)}\left(i s_{2} R\right)}\left(C_{1 n}^{\prime \prime} \cos n \eta+D_{1 n}^{\prime \prime} \sin n \eta\right)$,

(9)

where $E_{n}, M_{n}, \ldots$ are the sought coefficients, $H_{n}^{(1)}(z)$ is the Hankel's function with the index $n$. Taking into account (9), we can write the particular solutions of equations $\Delta \vartheta_{0}=\vartheta$, $\Delta \psi_{0}=\psi$ in the form

$\vartheta_{0}(\mathbf{x})=\frac{E_{0} \rho^{2}}{8}-$

$\frac{\rho^{2}}{4} \sum_{n=1}^{\infty} \frac{1}{n-1}\left(\frac{R}{\rho}\right)^{n}\left(E_{n} \cos n \eta+M_{n} \sin n \eta\right)$

(10)

$\psi_{0}(\mathbf{x})=\frac{A_{0}^{\prime} \rho^{2}}{8}-$

$\frac{\rho^{2}}{4} \sum_{n=1}^{\infty} \frac{1}{n-1}\left(\frac{R}{\rho}\right)^{n}\left(A_{n}^{\prime} \cos n \eta+B_{n}^{\prime} \sin n \eta\right)$.

We introduce the following notations:

$$
\begin{aligned}
& (\boldsymbol{x} \cdot \boldsymbol{f})^{-}=h_{1}^{-}, \quad(\operatorname{div} \boldsymbol{f})^{-}=h_{2}^{-}, \quad(\boldsymbol{x} \cdot \boldsymbol{F})^{-}=h_{3}^{-}, \\
& (\operatorname{div} \boldsymbol{F})^{-}=h_{4}^{-}, \quad \theta=h_{5}^{-} .
\end{aligned}
$$

From (8), passing to the limit as $\rho \rightarrow R$, to determine unknown functions, we obtain the following conditions

$$
\begin{aligned}
& \left(\mathbf{x} \cdot \frac{\partial \Psi}{\partial n}\right)=R \frac{\partial^{2}}{\partial \rho^{2}}\left[\frac{\lambda+\mu}{\mu} \psi_{0}-\frac{\beta}{\mu} \vartheta_{0}-\frac{\beta}{\mu_{0} s_{1}^{2}} \vartheta_{1}\right] \\
& +\left[\frac{\mu_{0} \psi-\beta \vartheta}{R}-h_{2}\right] \frac{R^{2}}{2 \mu}=G_{4}, \\
& (\lambda+\mu) \psi-\frac{\mu \beta}{\mu_{0}} \vartheta_{1}-\beta \vartheta=\frac{R}{2}\left(h_{2}+\frac{h_{1}}{R^{2}}\right), \\
& \vartheta+\vartheta_{1}=h_{5}, \quad-\frac{k}{k_{1}} s_{1}^{2} \vartheta_{1}=h_{4}, \\
& (\mathbf{x} \cdot \mathbf{w})^{-}=R \frac{\partial}{\partial \rho}\left[\frac{k_{3}}{k_{2}} \vartheta+\frac{k^{*}}{k_{1}^{*}} \vartheta_{1}\right]+h_{3}=G_{3} .
\end{aligned}
$$

At first, from system (11), we find

$$
\begin{gathered}
\vartheta_{1}^{-}=-\frac{k_{1}}{k s_{1}^{2}} h_{4}^{-}=G_{1}, \quad \vartheta^{-}=h_{5}^{-}-G_{1}=G, \\
\psi^{-}=\frac{1}{\lambda+\mu}\left[\beta G-\frac{\mu \beta}{\mu_{0}} G_{1}+\frac{R}{2}\left(\frac{1}{R^{2}} h_{1}^{-}+h_{2}^{-}\right)\right]=G_{2} .
\end{gathered}
$$

On the other hand, for determining the unknown coefficients, from (9) it follows

$$
\frac{E_{0}}{2}+\sum_{n=1}^{\infty}\left(E_{n} \cos n \eta+M_{n} \sin n \eta\right)=G
$$

$\frac{A_{0}^{\prime}}{2}+\sum_{n=1}^{\infty}\left(A_{n}^{\prime} \cos n \eta+B_{n}^{\prime} \sin n \eta\right)=G_{2}$,

$\frac{C_{10}}{2}+\sum_{n=1}^{\infty}\left(C_{1 n} \cos n \eta+D_{1 n} \sin n \eta\right)=G_{1}$,

$\frac{C^{\prime}{ }_{10}}{2}+\sum_{n=1}^{\infty}\left(C_{1 n} \cos n \eta+D^{\prime}{ }_{1 n} \sin n \eta\right)=G_{3}$,

From here we get

$E_{n}=\frac{1}{\pi} \int_{0}^{2 \pi} G^{-}(\eta) \cos n \eta d \eta$,

$M_{n}=\frac{1}{\pi} \int_{0}^{2 \pi} G^{-}(\eta) \sin n \eta d \eta$,

$A_{n}^{\prime}=\frac{1}{\pi} \int_{0}^{2 \pi} G_{2}(\eta) \cos n \eta d \eta$

$B_{n}^{\prime}=\frac{1}{\pi} \int_{0}^{2 \pi} G_{2}(\eta) \sin n \eta d \eta$

$C_{1 n}=\frac{1}{\pi} \int_{0}^{2 \pi} G_{1}^{-}(\eta) \cos n \eta d \eta$

$D_{1 n}=\frac{1}{\pi} \int_{0}^{2 \pi} G_{1}^{-}(\eta) \sin n \eta d \eta$

$C_{1 n}^{\prime}=\frac{1}{\pi} \int_{0}^{2 \pi} G_{3}^{-}(\eta) \cos n \eta d \eta$

$D_{1 n}^{\prime}=\frac{1}{\pi} \int_{0}^{2 \pi} G_{3}^{-}(\eta) \sin n \eta d \eta$

Substituting (12) into (9) and (10), we determine the functions

$\vartheta(\mathbf{x})=\frac{1}{2 \pi} \int_{0}^{2 \pi}\left[1+2 \sum_{n=1}^{\infty}\left(\frac{R}{\rho}\right)^{n} \cos n\left(\eta-\eta_{0}\right)\right] G^{-}(\eta) d \eta$

$=\frac{-1}{2 \pi} \int_{0}^{2 \pi} \frac{\left(R^{2}-\rho^{2}\right) G^{-}(\eta) d \eta}{R^{2}-2 R \rho \cos \left(\eta-\eta_{0}\right)+\rho^{2}}$, 
$\psi(\mathbf{x})=\frac{1}{2 \pi} \int_{0}^{2 \pi}\left[1+2 \sum_{n=1}^{\infty}\left(\frac{R}{\rho}\right)^{n} \cos n\left(\eta-\eta_{0}\right)\right] G_{2}(\eta) d \eta$

$=\frac{-1}{2 \pi} \int_{0}^{2 \pi} \frac{\left(R^{2}-\rho^{2}\right) G_{2}(\eta) d \eta}{R^{2}-2 R \rho \cos \left(\eta-\eta_{0}\right)+\rho^{2}}$

$(\mathbf{x} \cdot \mathbf{2})=\frac{1}{2 \pi} \int_{0}^{2 \pi} \frac{H_{0}^{(1)}\left(i s_{2} \rho\right)}{H_{0}^{(1)}\left(i s_{2} R\right)} G_{3}^{-}(\eta) d \eta$

$+\frac{1}{\pi} \int_{0}^{2 \pi} \sum_{n=1}^{\infty} \frac{H_{n}^{(1)}\left(i s_{2} \rho\right)}{H_{n}^{(1)}\left(i s_{2} R\right)} \cos n\left(\eta-\eta_{0}\right) G_{3}^{-}(\eta) d \eta$

$\vartheta_{1}(\mathbf{x})=\frac{1}{2 \pi} \int_{0}^{2 \pi} \frac{H_{0}^{(1)}\left(i s_{1} \rho\right)}{H_{0}^{(1)}\left(i s_{1} R\right)} G_{1}^{-}(\eta) d \eta$,

$+\frac{1}{\pi} \int_{0}^{2 \pi} \sum_{n=1}^{\infty} \frac{H_{n}^{(1)}\left(i s_{1} \rho\right)}{H_{n}^{(1)}\left(i s_{1} R\right)} \cos n\left(\eta-\eta_{0}\right) G_{1}^{-}(\eta) d \eta$,

$\mathbf{x}=\left(\rho, \eta_{0}\right) \in D$,

$\vartheta_{0}(\mathbf{x})=\frac{\rho^{2}}{8 \pi} \int_{0}^{2 \pi} G(\eta) d \eta$

$\frac{\rho^{2}}{4 \pi} \int_{0}^{2 \pi} \sum_{n=1}^{\infty} \frac{1}{n-1}\left(\frac{R}{\rho}\right)^{n} \cos n\left(\eta-\eta_{0}\right) G(\eta) d \eta$,

$\psi_{0}(\mathbf{x})=\frac{\rho^{2}}{8 \pi} \int_{0}^{2 \pi} G_{2}(\eta) d \eta$

$\frac{\rho^{2}}{4 \pi} \int_{0}^{2 \pi} \sum_{n=1}^{\infty} \frac{1}{n-1}\left(\frac{R}{\rho}\right)^{n} \cos n\left(\eta-\eta_{0}\right) G_{2}(\eta) d \eta$,

Thus, we have defined the functions

$\vartheta, \quad \vartheta_{0}, \quad \psi, \quad \psi_{0}, \quad \vartheta_{1}, \quad$ in the domain $D^{-}$

Now let us consider the following equation

$$
\left(\mathbf{x} \cdot \frac{\partial \Psi}{\partial n}\right)=\left(\frac{\partial}{\partial n}-\frac{1}{\rho}\right)(\mathbf{x} \cdot \Psi)=G_{4} .
$$

It is easily to verified, that the function $(\mathbf{x} \cdot \boldsymbol{\Psi})$, is the solutions of the following equation

$$
\Delta(\mathbf{x} \cdot \boldsymbol{\Psi})=2 \operatorname{div} \boldsymbol{\Psi}=2\left(\frac{\mu_{0}}{\mu} \psi-\frac{\beta}{\mu} \vartheta\right) .
$$

From here we find

$$
(\mathbf{x} \cdot \boldsymbol{\Psi})=\Omega+2\left(\frac{\mu_{0}}{\mu} \psi_{0}-\frac{\beta}{\mu} \vartheta_{0}\right),
$$

where $\Omega$ is an arbitrary harmonic function $\Delta \Omega=0$ and the functions $\vartheta_{0}, \quad$ and $\psi_{0}$ are chosen such that

$\Delta \vartheta_{0}=\vartheta, \quad \Delta \psi_{0}=\psi$.

Finally, for determining the function $\Omega$ we obtain the following Robin boundary value problem.

Find the harmonic function $\Omega$ in the domain $D^{-}$,

$\Delta \Omega=0, \quad x \in D^{-}$, satisfying the following boundary conditions on $S$ :

$\left(\frac{\partial \Omega}{\partial n}-\frac{\Omega}{R}\right)^{-}=G_{4}-\frac{2}{\mu}\left(\frac{\partial}{\partial n}-\frac{1}{\rho}\right)\left(\mu_{0} \psi_{0}-\beta \vartheta_{0}\right)=G_{5}^{-}$,

solution of which has the form

$\Omega(\mathbf{x})=\frac{1}{\pi} \int_{S} \ln \frac{r(\mathbf{x}, \mathbf{y})}{r(\mathbf{x})} g(y) d s+\frac{1}{\pi} \int_{S} g(y) d s$,

$r^{2}(x, y)=\left(x_{1}-y_{1}\right)^{2}+\left(x_{2}-y_{2}\right)^{2}$.

where $g(y)$ is a solution of the following Fredholm integral equation of second kind

$$
\begin{aligned}
& g(x)+\frac{1}{\pi} \int_{S} \frac{\partial}{\partial n_{x}} \ln \frac{r(x, y)}{r(x)} g(y) d s \\
& -\frac{1}{R \pi} \int_{S} \ln \frac{r(x, y)}{r(x)} g(y) d s-\frac{1}{R \pi} \int_{S} g(y) d s=G_{5}^{-} .
\end{aligned}
$$

It is well known that integral equation (14) is always solvable.

Thus, we have defined all unknown functions. Through inserting the obtained values into (6), we get the final form for solution of the considered Problem 1.

We assume that the functions $f_{j}, j=3,4, \quad \mathbf{f}, \mathbf{F}^{+}$, satisfy the following conditions on $S$

$$
f_{j} \in C^{3}(S) \quad j=3,4, \quad \mathbf{f}, \quad \mathbf{F}^{+} \in C^{3}(S) .
$$

Under these conditions the resulting series are absolutely and uniformly convergent.

Thus, the problem 1 is completely solved.

\section{Conclusions}

The main results of this work can be formulated as follows:

i. The regular solution $\mathbf{U}=(\mathbf{u}, \mathbf{w}, \theta)^{T}$ of equations (1)- (3) is obtained.

ii. Explicit solution of problem 1 for a plane with circular hole is presented. The obtained solutions are given by means of the 
harmonic, bi-harmonic and meta-harmonic functions. For the harmonic functions $\vartheta(\mathbf{x})$ and $\psi(\mathbf{x})$ the Poisson type formulas are obtained. The bi-harmonic and metaharmonic functions are presented as absolutely and uniformly convergent series.The harmonic function $\Omega(\mathbf{x})$ is given by (13), where the function $g(y)$ is a solution of the Fredholm integral equation of second kind.

\section{Conflict of Interest}

The author declares that there is no conflict of interest regarding the publication of this paper.

\section{References}

[1.] Grot, R. A., Thermodynamics of a continuum with microtemperatyre. Int. J. Engng. Sci. 7 (1969) 801-814.

[2.] Ieşan, D., and Quintanilla R., On a theory of thermoelasticity with microtemperatures. J. Thermal Stresses 23 (2000) 199215.

[3.] Svanadze, M., Fundamental solutions of the equations of the theory of thermoelasticity with microtemperatures. J. Thermal Stresses 27(2004) 151-170.

[4.] Scalia, A., Svanadze, M., and Tracina, R., Basic theorems in the equilibrium theory of thermoelasticity with microtemperatures, J. Thermal Stresses 33 (2010) 721-753.

[5.] Ieşan, D., On a theory of micromorphic elastic solids with microtemperatures. J. Thermal Stresses 24 (2001) 737-752.

[6.] Bitsadze, L., On one BVP for a thermo-microstretch elastic space with spherical cavity. Turkish. J. of Math. 42 (5) (2018) 2101 - 2111. DOI:10.3906/mat-1705-46

[7.] Bitsadze, L., Explicit solution of one boundary value problem of thermoelasticity for a circle with diffusion, microtemperatures and microconcentrations. Acta mechanica 231(9) (2020) 3551 3563.doi.org/10.1007/s00707-020-02729-0,

[8.] Bitsadze, L., Explicit solutions of the BVPs of the theory of thermoelasticity for an elastic circle with voids and microtemperatures(ZAMM). Journal of Applied Mathematics and Mechanics. 100(10) (2020).doi.org/10.1002/zamm.201800303

[9.] Bitsadze, L, Jaiani, G., Some basic boundary value problems of the plane thermoelasticity with microtemperatures, Math. Meth. Appl. Sci., 36 (2013) 956-966.

[10.] Bitsadze, L., Effective solution of the Dirichlet BVP of the linear theory of thermoelasticity with microtemperatures for a spherical ring. J. Thermal Stresses 36 (2013) 714-726.

[11.] Jaiani, G. Bitsadze, L., Basic Problems of Thermoelasticity with microtemperatures in the half-space. J.Thermal Stresses 41(9) (2018) 1101-1114.

[12.] Bitsadze, L., The Dirichlet BVP of the theory of thermoelasticity with microtemperatures for microstretch sphere. J. Thermal Stresses 39 (2016) 1074-1083.

[13.] Jaiani, G., Bitsadze, L., On basic problems for elastic prismatic shells with microtemperatures. ZAMM-Z. Angew. Math. Mech. 96 (2016) 1082-1088.

[14.] Riha, P., On the theory of heat conducting micropolar fluids with microtemperatures. Acta Mech. 23 (1975) 1-8.

[15.] Ieşan, D., Quintanilla, R., On thermoelastic bodies with inner structure and microtemperatures. J. Math. Anal. Appl., 354 (2009) 12-23.

[16.] Ieşan, D., Thermoelasticity of bodies with microstructure and microtemperatures. Int. J. Solids Struct. 44 (2007) 8648-8662.

[17.] Eringen, A.C., Microcontinuum field theories I, Foundations and Solids, Springer, New York, Berlin, Heidelberg,1999.

[18.] Casas, P.S.and Quintanilla, R., Exponential stability in thermoelasticity with microtemperatures. Int. J. Eng, Sci.,43 (2005),33-47.
[19.] Ciarletta, M., Tibullo, V., On the propagation of Rayleigh waves in a strongly ellipticthermoelastic material with microtemperatures. J. Thermal Stresses 39 (2016) 1111-1118.

[20.] Steeb, H., Singh, J.,Tomar, S.K., Time harmonic waves in thermoelastic materials with Microtemperatures. Mechanics Research Communications 48(2013) 8-18.

[21.] Ciarletta, S. M., Passarella, F., Tibullo, V., Plane harmonic waves in strongly elliptic thermoelastic materials with microtemperatures. J. Math. Anal. Appl. 424(2) (2015) 1182-1197

[22.] Chirita, S. Ciarletta, M., D'Apice, C., On the theory of thermoelasticity with Microtemperatures. J. of Math.Analysis and Applications.397(2013) 349-361.

[23.] Svanadze, M., Boundary value problems of the theory of thermoelasticity with microtemperaturesc. Proc. Appl.Math.mech. 3(2003) 188-189.

[24.] Singh, B., Propagation of Rayleingh wave in a thermoelastic solid half-space with microtemperatures.Int.J.Geophys.2014(2014),1-6.

[25.] Othman,M.I.A. Tantawi,R.S.Abd-Elazis,E.M.,Effect of initial stress on a thermoelastic medium voids and microtemperatures.J.of Porous Media (19)(2)(2016)155-172.

[26.] Othman,M.I.A. Abd-Elazis,E.M., Effect of initial stress and Hall current on magneto- thermoelastic porous medium with microtemperatures.Indian J.Phys.93(2019)476-485.

[27.] [27.]Aouadi,M., Ciarletta, M., Tibullo, V.,A thermoelastic diffusion theory with microtemperatures and microconcentrations. J. Thermal Stresses 40(2017)486-501.

[28.] Bazarra, N., Campo,M.,Fernandez,J.R., A thermoelastic problem with diffusion, with microtemperatures and microconcentrations. Acta Mech.230(2019) 31-48.

[29.] Ciarletta, M., Chirita,S. Some non-standard problems related with the mathematical model of thermoelasticity with microtemperatures. J. Thermal Stresses 36(2013)517-536.

[30.] Aouadi,M., Some theorems in the isotropic theory of microstretch of thermoelasticity with microtemperatures. J. Thermal Stresses 31(2008) 649-662.

\section{How to Cite This Article}

Bitsadze, L., The Neumann Type Boundary Value Problem in the theory of Thermoelasticity with Microtemperatures for a Plane with Circular Hole, Journal of Nature, Science \& Technology, 3 (2021), 1116. https://doi.org/10.36937/janset.2021.003.003 